\title{
Remarks on some inequalities for $s$-convex functions and applications
}

\section{Zheng Liu*}

\section{"Correspondence: lewzheng@163.ne \\ Institute of Applied Mathematics, School of Science, University of Science and Technology Liaoning, Anshan, 114051 Liaoning, China}

\begin{abstract}
Some new inequalities for functions whose second derivatives in absolute value at certain powers are s-convex in the second sense are established. Two mistakes in a recently published paper are pointed out and corrected.
\end{abstract}

MSC: 26D15

Keywords: convex function; s-convex function; Hölder inequality

\section{Introduction}

It is well known that a function $f: I \rightarrow \mathbf{R}, \emptyset \neq I \subset \mathbf{R}$ is called convex if

$$
f(\lambda x+(1-\lambda) y) \leq \lambda f(x)+(1-\lambda) f(y)
$$

holds for all $x, y \in I$ and $\lambda \in[0,1]$. If $-f: I \rightarrow \mathbf{R}$ is convex, then we say that $f: I \rightarrow \mathbf{R}$ is concave.

In [1], the class of $s$-convex function in the second sense is defined in the following way: a function $f:[0, \infty) \rightarrow \mathbf{R}$ is said to be $s$-convex in the second sense if

$$
f(\lambda x+(1-\lambda) y) \leq \lambda^{s} f(x)+(1-\lambda)^{s} f(y)
$$

holds for all $x, y \in[0, \infty), \lambda \in[0,1]$ and for some fixed $s \in(0,1]$. The class of $s$-convex functions in the second sense is usually denoted by $K_{s}^{2}$. It can be easily seen that for $s=1$ $s$-convexity reduces to ordinary convexity of functions defined on $[0, \infty)$. It is proved in [1] that all functions from $K_{s}^{2}, s \in(0,1)$ are nonnegative. Similarly, a function $f:[0, \infty) \rightarrow \mathbf{R}$ is said to be $s$-concave in the second sense for some fixed $s \in(0,1]$ if $-f \in K_{s}^{2}$. Thus we can conclude that an $s$-concave function is always nonpositive for any $s \in(0,1)$.

Example 1 [1] Let $s \in(0,1)$ and $a, b, c, \in \mathbf{R}$. Define the function $f:[0, \infty) \rightarrow \mathbf{R}$ as

$$
f(t)= \begin{cases}a, & t=0 \\ b t^{s}+c, & t>0 .\end{cases}
$$

It can be easily checked that

(i) if $b \geq 0$ and $0 \leq c \leq a$, then $f \in K_{s}^{2}$,

(ii) if $b>0$ and $c<0$, then $f \notin K_{s}^{2}$.

(c) 2015 Liu. This article is distributed under the terms of the Creative Commons Attribution 4.0 International License (http://creativecommons.org/licenses/by/4.0/), which permits unrestricted use, distribution, and reproduction in any medium, provided you give appropriate credit to the original author(s) and the source, provide a link to the Creative Commons license, and indicate if changes were made. 
Along this paper, we consider a real interval $I \subset \mathbf{R}$ and denote that $I^{\circ}$ is the interior of $I$. In a recent paper [2], Özdemir et al. proved the following inequalities for functions whose second derivatives in absolute value at certain powers are $s$-convex in the second sense.

Theorem 1 ([2], Theorem 2) Let $I \subset[0, \infty), f: I \rightarrow \mathbf{R}$ be a twice differentiable function on $I^{\circ}$ such that $f^{\prime \prime} \in L^{1}[a, b]$, where $a, b \in I$ with $a<b$. If $\left|f^{\prime \prime}\right|$ is s-convex in the second sense on $[a, b]$ for some fixed $s \in(0,1]$, then the following inequality holds:

$$
\begin{aligned}
& \left|\frac{1}{b-a} \int_{a}^{b} f(t) d t-f\left(\frac{a+b}{2}\right)\right| \\
& \quad \leq \frac{(b-a)^{2}}{8(s+1)(s+2)(s+3)}\left[\left|f^{\prime \prime}(a)\right|+(s+1)(s+2)\left|f^{\prime \prime}\left(\frac{a+b}{2}\right)\right|+\left|f^{\prime \prime}(b)\right|\right] \\
& \quad \leq \frac{\left[1+(s+2) 2^{1-s}\right](b-a)^{2}}{8(s+1)(s+2)(s+3)}\left[\left|f^{\prime \prime}(a)\right|+\left|f^{\prime \prime}(b)\right|\right] .
\end{aligned}
$$

Theorem 2 ([2], Theorem 4) Let $I \subset[0, \infty), f: I \rightarrow \mathbf{R}$ be a twice differentiable function on $I^{\circ}$ such that $f^{\prime \prime} \in L^{1}[a, b]$, where $a, b \in I$ with $a<b$. If $\left|f^{\prime \prime}\right|^{q}$ is s-convex in the second sense on $[a, b]$ for some fixed $s \in(0,1]$ and $q \geq 1$, then the following inequality holds:

$$
\begin{aligned}
& \left|\frac{1}{b-a} \int_{a}^{b} f(t) d t-f\left(\frac{a+b}{2}\right)\right| \\
& \leq \frac{(b-a)^{2}}{16}\left(\frac{1}{3}\right)^{1-\frac{1}{q}}\left\{\left(\frac{2}{(s+1)(s+2)(s+3)}\left|f^{\prime \prime}(a)\right|^{q}+\frac{1}{s+3}\left|f^{\prime \prime}\left(\frac{a+b}{2}\right)\right|^{q}\right)^{\frac{1}{q}}\right. \\
& \left.\quad+\left(\frac{1}{s+3}\left|f^{\prime \prime}\left(\frac{a+b}{2}\right)\right|^{q}+\frac{2}{(s+1)(s+2)(s+3)}\left|f^{\prime \prime}(b)\right|^{q}\right)^{\frac{1}{q}}\right\} .
\end{aligned}
$$

However, it is a pity that Theorem 5 in [2] is not valid since nonnegative $\left|f^{\prime \prime}\right|^{q}$ could not be an $s$-concave function for any fixed $s \in(0,1)$ which has been mentioned in [3], and it is the Hölder inequality but not the power mean inequality that has been used in proving Theorem 4 of [2].

In this work, we will first derive a new general inequality for functions whose second derivatives in absolute value at certain powers are $s$-convex in the second sense, which not only provides generalization of Theorem 1 and Theorem 2 but also gives some other interesting special results. Then we establish another new general inequality for functions whose second derivatives in absolute value at certain powers are $s$-convex in the second sense, which also gives some interesting special results. Finally, applications to some special means of real numbers are considered.

\section{Main results}

We first provide a new general inequality for functions whose second derivatives in absolute value at certain powers are $s$-convex in the second sense, and so we need the following lemma. 
Lemma 1 Let $I \subset \mathbf{R}, f: I \rightarrow \mathbf{R}$ be a twice differentiable function on $I^{\circ}$ such that $f^{\prime \prime} \in$ $L^{1}[a, b]$, where $a, b \in I$ with $a<b$. Then, for any $\theta \in[0,1]$, the following equality holds:

$$
\begin{gathered}
\frac{1}{b-a} \int_{a}^{b} f(t) d t-(1-\theta) f\left(\frac{a+b}{2}\right)-\theta \frac{f(a)+f(b)}{2} \\
=\frac{(b-a)^{2}}{16}\left[\int_{0}^{1}\left(t^{2}-2 \theta t\right) f^{\prime \prime}\left(t \frac{a+b}{2}+(1-t) a\right) d t\right. \\
\left.\quad+\int_{0}^{1}\left(t^{2}-2 \theta t\right) f^{\prime \prime}\left(t \frac{a+b}{2}+(1-t) b\right) d t\right]
\end{gathered}
$$

Proof Integrating by parts, we have the following identity:

$$
\begin{aligned}
I_{1}= & \int_{0}^{1}\left(t^{2}-2 \theta t\right) f^{\prime \prime}\left(t \frac{a+b}{2}+(1-t) a\right) d t \\
= & \left.\left(t^{2}-2 \theta t\right) \frac{2}{b-a} f^{\prime}\left(t \frac{a+b}{2}+(1-t) a\right)\right|_{0} ^{1}-\frac{4}{b-a} \int_{0}^{1}(t-\theta) f^{\prime}\left(t \frac{a+b}{2}+(1-t) a\right) d t \\
= & \frac{2(1-2 \theta)}{b-a} f^{\prime}\left(\frac{a+b}{2}\right)-\frac{4}{b-a}\left[\left.\frac{2(t-\theta)}{b-a} f\left(t \frac{a+b}{2}+(1-t) a\right)\right|_{0} ^{1}\right. \\
& \left.-\frac{2}{b-a} \int_{0}^{1} f\left(t \frac{a+b}{2}+(1-t) a\right) d t\right] \\
= & \frac{2(1-2 \theta)}{b-a} f^{\prime}\left(\frac{a+b}{2}\right)-\frac{8(1-\theta)}{(b-a)^{2}} f\left(\frac{a+b}{2}\right)-\frac{8 \theta}{(b-a)^{2}} f(a) \\
& +\frac{8}{(b-a)^{2}} \int_{0}^{1} f\left(t \frac{a+b}{2}+(1-t) a\right) d t .
\end{aligned}
$$

Using the change of variable $x=t \frac{a+b}{2}+(1-t) a$ for $t \in[0,1]$ and multiplying both sides of (4) by $\frac{(b-a)^{2}}{16}$, we obtain

$$
\begin{aligned}
& \frac{(b-a)^{2}}{16} \int_{0}^{1}\left(t^{2}-2 \theta t\right) f^{\prime \prime}\left(t \frac{a+b}{2}+(1-t) a\right) d t \\
& \quad=\frac{b-a}{8}(1-2 \theta) f^{\prime}\left(\frac{a+b}{2}\right)-\frac{1-\theta}{2} f\left(\frac{a+b}{2}\right)-\frac{\theta}{2} f(a)+\frac{1}{b-a} \int_{a}^{\frac{a+b}{2}} f(x) d x .
\end{aligned}
$$

Similarly, we observe that

$$
\begin{aligned}
& \frac{(b-a)^{2}}{16} \int_{0}^{1}\left(t^{2}-2 \theta t\right) f^{\prime \prime}\left(t \frac{a+b}{2}+(1-t) b\right) d t \\
& \quad=-\frac{b-a}{8}(1-2 \theta) f^{\prime}\left(\frac{a+b}{2}\right)-\frac{1-\theta}{2} f\left(\frac{a+b}{2}\right)-\frac{\theta}{2} f(b)+\frac{1}{b-a} \int_{\frac{a+b}{2}}^{b} f(x) d x .
\end{aligned}
$$

Thus, adding (5) and (6), we get the required identity (3).

Theorem 3 Let $I \subset[0, \infty), f: I \rightarrow \mathbf{R}$ be a twice differentiable function on $I^{\circ}$ such that $f^{\prime \prime} \in L^{1}[a, b]$, where $a, b \in I$ with $a<b$. If $\left|f^{\prime \prime}\right|^{q}$ is s-convex in the second sense on $[a, b]$ for 
some fixed $s \in(0,1]$ and $q \geq 1$, then the following inequalities hold:

$$
\begin{aligned}
& \left|\frac{1}{b-a} \int_{a}^{b} f(x) d x-(1-\theta) f\left(\frac{a+b}{2}\right)-\theta \frac{f(a)+f(b)}{2}\right| \\
& \leq \frac{(b-a)^{2}}{16}\left(\frac{8 \theta^{3}-3 \theta+1}{3}\right)^{1-\frac{1}{q}}\left\{\left[\frac{2(2 \theta)^{s+3}-2(s+3) \theta+s+2}{(s+2)(s+3)}\left|f^{\prime \prime}\left(\frac{a+b}{2}\right)\right|^{q}\right.\right. \\
& \left.\quad+\frac{4(1-2 \theta)^{s+2}[(s+1) \theta+1]+2(s+3) \theta-2}{(s+1)(s+2)(s+3)}\left|f^{\prime \prime}(a)\right|^{q}\right]^{\frac{1}{q}} \\
& +\left[\frac{2(2 \theta)^{s+3}-2(s+3) \theta+s+2}{(s+2)(s+3)}\left|f^{\prime \prime}\left(\frac{a+b}{2}\right)\right|^{q}\right. \\
& \left.\left.+\frac{4(1-2 \theta)^{s+2}[(s+1) \theta+1]+2(s+3) \theta-2}{(s+1)(s+2)(s+3)}\left|f^{\prime \prime}(b)\right|^{q}\right]^{\frac{1}{q}}\right\}
\end{aligned}
$$

for $0 \leq \theta \leq \frac{1}{2}$ and

$$
\begin{aligned}
& \left|\frac{1}{b-a} \int_{a}^{b} f(x) d x-(1-\theta) f\left(\frac{a+b}{2}\right)-\theta \frac{f(a)+f(b)}{2}\right| \\
& \leq \frac{(b-a)^{2}}{16}\left(\theta-\frac{1}{3}\right)^{1-\frac{1}{q}}\left\{\left[\frac{2(s+3) \theta-s-2}{(s+2)(s+3)}\left|f^{\prime \prime}\left(\frac{a+b}{2}\right)\right|^{q}\right.\right. \\
& \left.\quad+\frac{2(s+3) \theta-2}{(s+1)(s+2)(s+3)}\left|f^{\prime \prime}(a)\right|^{q}\right]^{\frac{1}{q}}+\left[\frac{2(s+3) \theta-s-2}{(s+2)(s+3)}\left|f^{\prime \prime}\left(\frac{a+b}{2}\right)\right|^{q}\right. \\
& \left.\left.\quad+\frac{2(s+3) \theta-2}{(s+1)(s+2)(s+3)}\left|f^{\prime \prime}(b)\right|^{q}\right]^{\frac{1}{q}}\right\}
\end{aligned}
$$

for $\frac{1}{2} \leq \theta \leq 1$.

Proof In case $0 \leq \theta \leq \frac{1}{2}$, by Lemma 1 and using the Hölder inequality, we have

$$
\begin{aligned}
& \left|\frac{1}{b-a} \int_{a}^{b} f(t) d t-(1-\theta) f\left(\frac{a+b}{2}\right)-\theta \frac{f(a)+f(b)}{2}\right| \\
& \leq \frac{(b-a)^{2}}{16}\left[\int_{0}^{1}\left|t^{2}-2 \theta t\right|\left|f^{\prime \prime}\left(t \frac{a+b}{2}+(1-t) a\right)\right| d t\right. \\
& \left.+\int_{0}^{1}\left|t^{2}-2 \theta t\right|\left|f^{\prime \prime}\left(t \frac{a+b}{2}+(1-t) b\right)\right| d t\right] \\
& \leq \frac{(b-a)^{2}}{16}\left[\left(\int_{0}^{1}\left|t^{2}-2 \theta t\right| d t\right)^{1-\frac{1}{q}}\left(\int_{0}^{1}\left|t^{2}-2 \theta t\right|\left|f^{\prime \prime}\left(t \frac{a+b}{2}+(1-t) a\right)\right|^{q} d t\right)^{\frac{1}{q}}\right. \\
& \left.+\left(\int_{0}^{1}\left|t^{2}-2 \theta t\right| d t\right)^{1-\frac{1}{q}}\left(\int_{0}^{1}\left|t^{2}-2 \theta t\right|\left|f^{\prime \prime}\left(t \frac{a+b}{2}+(1-t) b\right)\right|^{q} d t\right)^{\frac{1}{q}}\right] \\
& \leq \frac{(b-a)^{2}}{16}\left[( \frac { 8 \theta ^ { 3 } - 3 \theta + 1 } { 3 } ) ^ { 1 - \frac { 1 } { q } } \left(\int _ { 0 } ^ { 1 } | t ^ { 2 } - 2 \theta t | \left(t^{s}\left|f^{\prime \prime}\left(\frac{a+b}{2}\right)\right|^{q}\right.\right.\right. \\
& \left.\left.+(1-t)^{s}\left|f^{\prime \prime}(a)\right|^{q}\right) d t\right)^{\frac{1}{q}}
\end{aligned}
$$




$$
\begin{aligned}
& \left.+\left(\frac{8 \theta^{3}-3 \theta+1}{3}\right)^{1-\frac{1}{q}}\left(\int_{0}^{1}\left|t^{2}-2 \theta t\right|\left(t^{s}\left|f^{\prime \prime}\left(\frac{a+b}{2}\right)\right|^{q}+(1-t)^{s}\left|f^{\prime \prime}(b)\right|^{q}\right) d t\right)^{\frac{1}{q}}\right] \\
= & \frac{(b-a)^{2}}{16}\left[( \frac { 8 \theta ^ { 3 } - 3 \theta + 1 } { 3 } ) ^ { 1 - \frac { 1 } { q } } \left(\int _ { 0 } ^ { 2 \theta } t ( 2 \theta - t ) \left(t^{s}\left|f^{\prime \prime}\left(\frac{a+b}{2}\right)\right|^{q}\right.\right.\right. \\
& \left.\left.+(1-t)^{s}\left|f^{\prime \prime}(a)\right|^{q}\right) d t+\int_{2 \theta}^{1} t(t-2 \theta)\left(t^{s}\left|f^{\prime \prime}\left(\frac{a+b}{2}\right)\right|^{q}+(1-t)^{s}\left|f^{\prime \prime}(a)\right|^{q}\right) d t\right)^{\frac{1}{q}} \\
& +\left(\frac{8 \theta^{3}-3 \theta+1}{3}\right)^{1-\frac{1}{q}}\left(\int_{0}^{2 \theta} t(2 \theta-t)\left(t^{s}\left|f^{\prime \prime}\left(\frac{a+b}{2}\right)\right|^{q}+(1-t)^{s}\left|f^{\prime \prime}(b)\right|^{q}\right) d t\right. \\
& \left.\left.+\int_{2 \theta}^{1} t(t-2 \theta)\left(t^{s}\left|f^{\prime \prime}\left(\frac{a+b}{2}\right)\right|^{q}+(1-t)^{s}\left|f^{\prime \prime}(b)\right|^{q}\right) d t\right)^{\frac{1}{q}}\right] \\
= & \frac{(b-a)^{2}}{16}\left(\frac{8 \theta^{3}-3 \theta+1}{3}\right)^{1-\frac{1}{q}}\left\{\left[\frac{2(2 \theta)^{s+3}-2(s+3) \theta+s+2}{(s+2)(s+3)}\left|f^{\prime \prime}\left(\frac{a+b}{2}\right)\right|^{q}\right.\right. \\
& \left.+\frac{4(1-2 \theta)^{s+2}[(s+1) \theta+1]+2(s+3) \theta-2}{(s+1)(s+2)(s+3)}\left|f^{\prime \prime}(a)\right|^{q}\right]^{\frac{1}{q}} \\
& +\left[\frac{2(2 \theta)^{s+3}-2(s+3) \theta+s+2}{(s+2)(s+3)}\left|f^{\prime \prime}\left(\frac{a+b}{2}\right)\right|^{q}\right. \\
& \left.\left.+\frac{4(1-2 \theta)^{s+2}[(s+1) \theta+1]+2(s+3) \theta-2}{(s+1)(s+2)(s+3)}\left|f^{\prime \prime}(b)\right|^{q}\right]^{\frac{1}{q}}\right\},
\end{aligned}
$$

where

$$
\begin{aligned}
& \int_{0}^{1}\left|t^{2}-2 \theta t\right| d t=\int_{0}^{2 \theta} t(2 \theta-t) d t+\int_{2 \theta}^{1} t(t-2 \theta) d t=\frac{8 \theta^{3}-3 \theta+1}{3}, \\
& \int_{0}^{2 \theta} t^{s+1}(2 \theta-t) d t=\frac{(2 \theta)^{s+3}}{(s+2)(s+3)} \\
& \int_{2 \theta}^{1} t^{s+1}(t-2 \theta) d t=\frac{(2 \theta)^{s+3}-2(s+3) \theta+s+2}{(s+2)(s+3)} \\
& \int_{0}^{2 \theta} t(1-t)^{s}(2 \theta-t) d t=\frac{(1-2 \theta)^{s+2}[2(s+1) \theta+2]+2(s+3) \theta-2}{(s+1)(s+2)(s+3)}
\end{aligned}
$$

and

$$
\int_{2 \theta}^{1} t(1-t)^{s}(t-2 \theta) d t=\frac{(1-2 \theta)^{s+2}[2(s+1) \theta+2]}{(s+1)(s+2)(s+3)} .
$$

In case $\frac{1}{2} \leq \theta \leq 1$, by Lemma 1 and using the Hölder inequality, we have

$$
\begin{aligned}
\left|\frac{1}{b-a} \int_{a}^{b} f(t) d t-(1-\theta) f\left(\frac{a+b}{2}\right)-\theta \frac{f(a)+f(b)}{2}\right| \\
\leq \frac{(b-a)^{2}}{16}\left[\int_{0}^{1}\left|t^{2}-2 \theta t\right|\left|f^{\prime \prime}\left(t \frac{a+b}{2}+(1-t) a\right)\right| d t\right. \\
\left.\quad+\int_{0}^{1}\left|t^{2}-2 \theta t\right|\left|f^{\prime \prime}\left(t \frac{a+b}{2}+(1-t) b\right)\right| d t\right]
\end{aligned}
$$




$$
\begin{aligned}
& \leq \frac{(b-a)^{2}}{16}\left[\left(\int_{0}^{1}\left|t^{2}-2 \theta t\right| d t\right)^{1-\frac{1}{q}}\left(\int_{0}^{1}\left|t^{2}-2 \theta t\right|\left|f^{\prime \prime}\left(t \frac{a+b}{2}+(1-t) a\right)\right|^{q} d t\right)^{\frac{1}{q}}\right. \\
& \left.+\left(\int_{0}^{1}\left|t^{2}-2 \theta t\right| d t\right)^{1-\frac{1}{q}}\left(\int_{0}^{1}\left|t^{2}-2 \theta t\right|\left|f^{\prime \prime}\left(t \frac{a+b}{2}+(1-t) b\right)\right|^{q} d t\right)^{\frac{1}{q}}\right] \\
& \leq \frac{(b-a)^{2}}{16}\left\{\left(\theta-\frac{1}{3}\right)^{1-\frac{1}{q}}\left(\int_{0}^{1}\left|t^{2}-2 \theta t\right|\left[t^{s}\left|f^{\prime \prime}\left(\frac{a+b}{2}\right)\right|^{q}+(1-t)^{s}\left|f^{\prime \prime}(a)\right|^{q}\right] d t\right)^{\frac{1}{q}}\right. \\
& \left.+\left(\theta-\frac{1}{3}\right)^{1-\frac{1}{q}}\left(\int_{0}^{1}\left|t^{2}-2 \theta t\right|\left[t^{s}\left|f^{\prime \prime}\left(\frac{a+b}{2}\right)\right|^{q}+(1-t)^{s}\left|f^{\prime \prime}(b)\right|^{q}\right] d t\right)^{\frac{1}{q}}\right\} \\
& =\frac{(b-a)^{2}}{16}\left(\theta-\frac{1}{3}\right)^{1-\frac{1}{q}}\left\{\left[\left(\int_{0}^{1} t^{s+1}(2 \theta-t) d t\right)\left|f^{\prime \prime}\left(\frac{a+b}{2}\right)\right|^{q}\right.\right. \\
& \left.+\left(\int_{0}^{1} t(1-t)^{s}(2 \theta-t) d t\right)\left|f^{\prime \prime}(a)\right|^{q}\right]^{\frac{1}{q}} \\
& \left.+\left[\left(\int_{0}^{1} t^{s+1}(2 \theta-t) d t\right)\left|f^{\prime \prime}\left(\frac{a+b}{2}\right)\right|^{q}+\left(\int_{0}^{1} t(1-t)^{s}(2 \theta-t) d t\right)\left|f^{\prime \prime}(b)\right|^{q}\right]^{\frac{1}{q}}\right\} \\
& =\frac{(b-a)^{2}}{16}\left(\theta-\frac{1}{3}\right)^{1-\frac{1}{q}}\left\{\left[\frac{2(s+3) \theta-s-2}{(s+2)(s+3)}\left|f^{\prime \prime}\left(\frac{a+b}{2}\right)\right|^{q}\right.\right. \\
& \left.+\frac{2(s+3) \theta-2}{(s+1)(s+2)(s+3)}\left|f^{\prime \prime}(a)\right|^{q}\right]^{\frac{1}{q}}+\left[\frac{2(s+3) \theta-s-2}{(s+2)(s+3)}\left|f^{\prime \prime}\left(\frac{a+b}{2}\right)\right|^{q}\right. \\
& \left.\left.+\frac{2(s+3) \theta-2}{(s+1)(s+2)(s+3)}\left|f^{\prime \prime}(b)\right|^{q}\right]^{\frac{1}{q}}\right\}
\end{aligned}
$$

where

$$
\begin{aligned}
& \int_{0}^{1}\left|t^{2}-2 \theta t\right| d t=\int_{0}^{1} t(2 \theta-t) d t=\theta-\frac{1}{3} \\
& \int_{0}^{1} t^{s+1}(2 \theta-t) d t=\frac{2(s+3) \theta-s-2}{(s+2)(s+3)}
\end{aligned}
$$

and

$$
\int_{0}^{1} t(1-t)^{s}(2 \theta-t) d t=\frac{2(s+3) \theta-2}{(s+1)(s+2)(s+3)} .
$$

The proof is thus completed.

$\operatorname{Remark} 1$ If we take $\theta=0$ in (7), then we get a midpoint type inequality

$$
\begin{aligned}
& \left|\frac{1}{b-a} \int_{a}^{b} f(t) d t-f\left(\frac{a+b}{2}\right)\right| \\
& \leq \frac{(b-a)^{2}}{16}\left(\frac{1}{3}\right)^{1-\frac{1}{q}}\left\{\left[\frac{1}{s+3}\left|f^{\prime \prime}\left(\frac{a+b}{2}\right)\right|^{q}+\frac{2}{(s+1)(s+2)(s+3)}\left|f^{\prime \prime}(a)\right|^{q}\right]^{\frac{1}{q}}\right. \\
& \left.+\left[\frac{1}{s+3}\left|f^{\prime \prime}\left(\frac{a+b}{2}\right)\right|^{q}+\frac{2}{(s+1)(s+2)(s+3)}\left|f^{\prime \prime}(b)\right|^{q}\right]^{\frac{1}{q}}\right\} .
\end{aligned}
$$


If we take $\theta=1$ in (8), then we get a trapezoid type inequality

$$
\begin{aligned}
& \left|\frac{1}{b-a} \int_{a}^{b} f(t) d t-\frac{f(a)+f(b)}{2}\right| \\
& \leq \frac{(b-a)^{2}}{16}\left(\frac{2}{3}\right)^{1-\frac{1}{q}}\left\{\left[\frac{s+4}{(s+2)(s+3)}\left|f^{\prime \prime}\left(\frac{a+b}{2}\right)\right|^{q}+\frac{2}{(s+1)(s+3)}\left|f^{\prime \prime}(a)\right|^{q}\right]^{\frac{1}{q}}\right. \\
& \left.\quad+\left[\frac{s+4}{(s+2)(s+3)}\left|f^{\prime \prime}\left(\frac{a+b}{2}\right)\right|^{q}+\frac{2}{(s+1)(s+3)}\left|f^{\prime \prime}(b)\right|^{q}\right]^{\frac{1}{q}}\right\} .
\end{aligned}
$$

If we take $\theta=\frac{1}{3}$ in (7), then we get a Simpson type inequality

$$
\begin{aligned}
& \left|\frac{1}{b-a} \int_{a}^{b} f(t) d t-\frac{1}{6}\left[f(a)+4 f\left(\frac{a+b}{2}\right)+f(b)\right]\right| \\
& \leq \frac{(b-a)^{2}}{16}\left(\frac{8}{81}\right)^{1-\frac{1}{q}}\left\{\left[\left(\frac{s}{3(s+2)(s+3)}+\frac{2}{(s+2)(s+3)}\left(\frac{2}{s+3}\right)^{s+3}\right)\left|f^{\prime \prime}\left(\frac{a+b}{2}\right)\right|^{q}\right.\right. \\
& \left.\quad+\left(\frac{4 s+16}{(s+1)(s+2)(s+3)}\left(\frac{1}{3}\right)^{s+3}+\frac{2 s}{3(s+1)(s+2)(s+3)}\right)\left|f^{\prime \prime}(a)\right|^{q}\right]^{\frac{1}{q}} \\
& \quad \times\left[\left(\frac{s}{3(s+2)(s+3)}+\frac{2}{(s+2)(s+3)}\left(\frac{2}{s+3}\right)^{s+3}\right)\left|f^{\prime \prime}\left(\frac{a+b}{2}\right)\right|^{q}\right. \\
& \left.\left.\quad+\left(\frac{4 s+16}{(s+1)(s+2)(s+3)}\left(\frac{1}{3}\right)^{s+3}+\frac{2 s}{3(s+1)(s+2)(s+3)}\right)\left|f^{\prime \prime}(b)\right|^{q}\right]^{\frac{1}{q}}\right\}
\end{aligned}
$$

If we take $\theta=\frac{1}{2}$ in (7) or (8), then we get an averaged midpoint-trapezoid type inequality

$$
\begin{aligned}
& \left|\frac{1}{b-a} \int_{a}^{b} f(t) d t-\frac{1}{4}\left[f(a)+2 f\left(\frac{a+b}{2}\right)+f(b)\right]\right| \\
& \leq \frac{(b-a)^{2}}{16}\left(\frac{1}{6}\right)^{1-\frac{1}{q}}\left\{\left[\frac{1}{(s+2)(s+3)}\left(\left|f^{\prime \prime}\left(\frac{a+b}{2}\right)\right|^{q}+\left|f^{\prime \prime}(a)\right|^{q}\right)\right]^{\frac{1}{q}}\right. \\
& \left.\quad+\left[\frac{1}{(s+2)(s+3)}\left(\left|f^{\prime \prime}\left(\frac{a+b}{2}\right)\right|^{q}+\left|f^{\prime \prime}(b)\right|^{q}\right)\right]^{\frac{1}{q}}\right\}
\end{aligned}
$$

Corollary 1 Let $I \subset[0, \infty), f: I \rightarrow \mathbf{R}$ be a twice differentiable function on $I^{\circ}$ such that $f^{\prime \prime} \in L^{1}[a, b]$, where $a, b \in I$ with $a<b$. If $\left|f^{\prime \prime}\right|$ is s-convex in the second sense on $[a, b]$ for some fixed $s \in(0,1]$, then the following inequalities hold:

$$
\begin{aligned}
& \left|\frac{1}{b-a} \int_{a}^{b} f(x) d x-(1-\theta) f\left(\frac{a+b}{2}\right)-\theta \frac{f(a)+f(b)}{2}\right| \\
& \leq \frac{(b-a)^{2}}{8}\left[\frac{2(2 \theta)^{s+3}-2(s+3) \theta+s+2}{(s+2)(s+3)}\left|f^{\prime \prime}\left(\frac{a+b}{2}\right)\right|\right. \\
& \left.\quad+\frac{2(1-2 \theta)^{s+2}[(s+1) \theta+1]+(s+3) \theta-1}{(s+1)(s+2)(s+3)}\left(\left|f^{\prime \prime}(a)\right|+\left|f^{\prime \prime}(b)\right|\right)\right]
\end{aligned}
$$




$$
\begin{aligned}
& \text { for } 0 \leq \theta \leq \frac{1}{2} \text { and } \\
& \left|\frac{1}{b-a} \int_{a}^{b} f(x) d x-(1-\theta) f\left(\frac{a+b}{2}\right)-\theta \frac{f(a)+f(b)}{2}\right| \\
& \leq \frac{(b-a)^{2}}{8}\left[\frac{2(s+3) \theta-s-2}{(s+2)(s+3)}\left|f^{\prime \prime}\left(\frac{a+b}{2}\right)\right|\right. \\
& \left.+\frac{(s+3) \theta-1}{(s+1)(s+2)(s+3)}\left(\left|f^{\prime \prime}(a)\right|+\left|f^{\prime \prime}(b)\right|\right)\right]
\end{aligned}
$$

for $\frac{1}{2} \leq \theta \leq 1$

Proof Inequalities (13) and (14) are immediate by setting $q=1$ in (7) and (8) of Theorem 3.

Remark 2 If we take $\theta=0$ in (13), then we get a midpoint type inequality

$$
\begin{aligned}
& \left|\frac{1}{b-a} \int_{a}^{b} f(t) d t-f\left(\frac{a+b}{2}\right)\right| \\
& \quad \leq \frac{(b-a)^{2}}{8}\left[\frac{1}{s+3}\left|f^{\prime \prime}\left(\frac{a+b}{2}\right)\right|+\frac{1}{(s+1)(s+2)(s+3)}\left(\left|f^{\prime \prime}(a)\right|+\left|f^{\prime \prime}(b)\right|\right)\right] .
\end{aligned}
$$

If we take $\theta=1$ in (14), then we get a trapezoid type inequality

$$
\begin{aligned}
& \left|\frac{1}{b-a} \int_{a}^{b} f(t) d t-\frac{f(a)+f(b)}{2}\right| \\
& \quad \leq \frac{(b-a)^{2}}{8}\left[\frac{s+4}{(s+2)(s+3)}\left|f^{\prime \prime}\left(\frac{a+b}{2}\right)\right|+\frac{1}{(s+1)(s+3)}\left(\left|f^{\prime \prime}(a)\right|+\left|f^{\prime \prime}(b)\right|\right)\right] .
\end{aligned}
$$

If we take $\theta=\frac{1}{3}$ in (13), then we get a Simpson type inequality

$$
\begin{aligned}
& \left|\frac{1}{b-a} \int_{a}^{b} f(t) d t-\frac{1}{6}\left[f(a)+4 f\left(\frac{a+b}{2}\right)+f(b)\right]\right| \\
& \leq \frac{(b-a)^{2}}{8}\left\{\left[\frac{s}{3(s+2)(s+3)}+\frac{2}{(s+2)(s+3)}\left(\frac{2}{3}\right)^{s+3}\right]\left|f^{\prime \prime}\left(\frac{a+b}{2}\right)\right|\right. \\
& \left.\quad+\left[\frac{2 s+8}{(s+1)(s+2)(s+3)}\left(\frac{1}{3}\right)^{s+3}+\frac{s}{3(s+1)(s+2)(s+3)}\right]\left(\left|f^{\prime \prime}(a)\right|+\left|f^{\prime \prime}(b)\right|\right)\right\} .
\end{aligned}
$$

If we take $\theta=\frac{1}{2}$ in (13) or (14), then we get an averaged midpoint-trapezoid type inequality

$$
\begin{aligned}
& \left|\frac{1}{b-a} \int_{a}^{b} f(t) d t-\frac{1}{4}\left[f(a)+2 f\left(\frac{a+b}{2}\right)+f(b)\right]\right| \\
& \quad \leq \frac{(b-a)^{2}}{8}\left[\frac{1}{(s+2)(s+3)}\left|f^{\prime \prime}\left(\frac{a+b}{2}\right)\right|+\frac{1}{2(s+2)(s+3)}\right]\left(\left|f^{\prime \prime}(a)\right|+\left|f^{\prime \prime}(b)\right|\right) .
\end{aligned}
$$


Remark 3 If we put $M=\sup _{x \in[a, b]}\left|f^{\prime \prime}\right|$ in (15)-(18), then we have

$$
\begin{aligned}
& \left|\frac{1}{b-a} \int_{a}^{b} f(t) d t-f\left(\frac{a+b}{2}\right)\right| \leq \frac{M\left(s^{2}+3 s+4\right)(b-a)^{2}}{8(s+1)(s+2)(s+3)}, \\
& \left|\frac{1}{b-a} \int_{a}^{b} f(t) d t-\frac{f(a)+f(b)}{2}\right| \leq \frac{M\left(s^{2}+7 s+8\right)(b-a)^{2}}{8(s+1)(s+2)(s+3)}, \\
& \left|\frac{1}{b-a} \int_{a}^{b} f(t) d t-\frac{1}{6}\left[f(a)+4 f\left(\frac{a+b}{2}\right)+f(b)\right]\right| \\
& \leq \frac{M(b-a)^{2}}{24(s+1)(s+2)(s+3)}\left[s^{2}+3 s+(4 s+16)\left(\frac{1}{3}\right)^{s+2}+6(s+1)\left(\frac{2}{3}\right)^{s+3}\right],
\end{aligned}
$$

and

$$
\left|\frac{1}{b-a} \int_{a}^{b} f(t) d t-\frac{1}{4}\left[f(a)+2 f\left(\frac{a+b}{2}\right)+f(b)\right]\right| \leq \frac{M(b-a)^{2}}{4(s+2)(s+3)}
$$

Remark 4 If we further take $s=1$ in (19)-(22), i.e., for functions $f$ with convex $\left|f^{\prime \prime}\right|$, we have

$$
\begin{aligned}
& \left|\frac{1}{b-a} \int_{a}^{b} f(t) d t-f\left(\frac{a+b}{2}\right)\right| \leq \frac{M(b-a)^{2}}{24}, \\
& \left|\frac{1}{b-a} \int_{a}^{b} f(t) d t-\frac{f(a)+f(b)}{2}\right| \leq \frac{M(b-a)^{2}}{12}, \\
& \left|\frac{1}{b-a} \int_{a}^{b} f(t) d t-\frac{1}{6}\left[f(a)+4 f\left(\frac{a+b}{2}\right)+f(b)\right]\right| \leq \frac{M(b-a)^{2}}{81} \\
& \left|\frac{1}{b-a} \int_{a}^{b} f(t) d t-\frac{1}{4}\left[f(a)+2 f\left(\frac{a+b}{2}\right)+f(b)\right]\right| \leq \frac{M(b-a)^{2}}{48}
\end{aligned}
$$

Obviously, (23)-(26) indicate that the Simpson type inequality has the best error estimation for functions $f$ with convex $\left|f^{\prime \prime}\right|$.

Now we turn to establish another new general inequality for functions whose second derivatives in absolute value at certain powers are $s$-convex in the second sense, we need the following lemma.

Lemma 2 Let $I \subset \mathbf{R}, f: I \rightarrow \mathbf{R}$ be a twice differentiable function on $I^{\circ}$ such that $f^{\prime \prime} \in$ $L^{1}[a, b]$, where $a, b \in I$ with $a<b$. Then, for any $\theta \in[0,1]$, the following equality holds:

$$
\begin{aligned}
& \frac{1}{b-a} \int_{a}^{b} f(t) d t-(1-\theta) f\left(\frac{a+b}{2}\right)-\theta \frac{f(a)+f(b)}{2} \\
& =(b-a)^{2} \int_{0}^{1} k(t) f^{\prime \prime}(t a+(1-t) b) d t
\end{aligned}
$$

where

$$
k(t)= \begin{cases}\frac{1}{2} t(t-\theta), & 0 \leq t \leq \frac{1}{2} \\ \frac{1}{2}(1-t)(1-\theta-t), & \frac{1}{2} \leq t \leq 1\end{cases}
$$


Proof See, e.g., Lemma 2 in [4].

Theorem 4 Let $I \subset[0, \infty), f: I \rightarrow \mathbf{R}$ be a twice differentiable function on $I^{\circ}$ such that $f^{\prime \prime} \in L^{1}[a, b]$, where $a, b \in I$ with $a<b$. If $\left|f^{\prime \prime}\right|^{q}$ is s-convex in the second sense on $[a, b]$ for some fixed $s \in(0,1]$ and $q \geq 1$, then the following inequalities hold:

$$
\begin{aligned}
& \left|\frac{1}{b-a} \int_{a}^{b} f(x) d x-(1-\theta) f\left(\frac{a+b}{2}\right)-\theta \frac{f(a)+f(b)}{2}\right| \\
& \leq \frac{(b-a)^{2}}{2}\left(\frac{8 \theta^{3}-3 \theta+1}{24}\right)^{1-\frac{1}{q}}\left\{\left[\frac{2 \theta^{s+3}}{(s+2)(s+3)}\right.\right. \\
& \left.\quad+\frac{2(1-\theta)^{s+2}[(s+1) \theta+2]+(s+3) \theta-2}{(s+1)(s+2)(s+3)}-\frac{1-\theta}{2^{s+1}(s+1)(s+2)}\right] \\
& \left.\quad \times\left(\left|f^{\prime \prime}(a)\right|^{q}+\left|f^{\prime \prime}(b)\right|^{q}\right)\right\}^{\frac{1}{q}}
\end{aligned}
$$

for $0 \leq \theta \leq \frac{1}{2}$ and

$$
\begin{aligned}
& \left|\frac{1}{b-a} \int_{a}^{b} f(x) d x-(1-\theta) f\left(\frac{a+b}{2}\right)-\theta \frac{f(a)+f(b)}{2}\right| \\
& \quad \leq \frac{(b-a)^{2}}{2}\left(\frac{3 \theta-1}{24}\right)^{1-\frac{1}{q}}\left\{\left[\frac{(s+3) \theta-2}{(s+1)(s+2)(s+3)}+\frac{1-\theta}{2^{s+1}(s+1)(s+2)}\right]\right. \\
& \left.\quad \times\left(\left|f^{\prime \prime}(a)\right|^{q}+\left|f^{\prime \prime}(b)\right|^{q}\right)\right\}^{\frac{1}{q}}
\end{aligned}
$$

for $\frac{1}{2} \leq \theta \leq 1$.

Proof In case $0 \leq \theta \leq \frac{1}{2}$, by Lemma 2 and using the Hölder inequality, we have

$$
\begin{aligned}
\left|\frac{1}{b-a} \int_{a}^{b} f(t) d t-(1-\theta) f\left(\frac{a+b}{2}\right)-\theta \frac{f(a)+f(b)}{2}\right| & \\
\leq & (b-a)^{2} \int_{0}^{1}|k(t)|\left|f^{\prime \prime}(t a+(1-t) b)\right| d t \\
\leq & (b-a)^{2}\left[\int_{0}^{1}|k(t)| d t\right]^{1-\frac{1}{q}}\left[\int_{0}^{1}|k(t)|\left|f^{\prime \prime}(t a+(1-t) b)\right|^{q} d t\right]^{\frac{1}{q}} \\
\leq & (b-a)^{2}\left(\frac{8 \theta^{3}-3 \theta+1}{24}\right)^{1-\frac{1}{q}}\left(\int_{0}^{1}|k(t)|\left[t^{s}\left|f^{\prime \prime}(a)\right|^{q}+(1-t)^{s}\left|f^{\prime \prime}(b)\right|^{q}\right] d t\right)^{\frac{1}{q}} \\
= & \frac{(b-a)}{2}\left(\frac{8 \theta^{3}-3 \theta+1}{24}\right)^{1-\frac{1}{q}} \\
\quad \times & {\left[\left(\int_{0}^{\frac{1}{2}} t^{s+1}|t-\theta| d t+\int_{\frac{1}{2}}^{1} t^{s}(1-t)|1-\theta-t| d t\right)\left|f^{\prime \prime}(a)\right|^{q}\right.} \\
& \left.+\left(\int_{0}^{\frac{1}{2}} t(1-t)^{s}|t-\theta| d t+\int_{\frac{1}{2}}^{1}(1-t)^{s+1}|1-\theta-t| d t\right)\left|f^{\prime \prime}(b)\right|^{q}\right]
\end{aligned}
$$




$$
\begin{aligned}
= & \frac{(b-a)}{2}\left(\frac{8 \theta^{3}-3 \theta+1}{24}\right)^{1-\frac{1}{q}}\left[\left(\int_{0}^{\theta} t^{s+1}(\theta-t) d t+\int_{\theta}^{\frac{1}{2}} t^{s+1}(t-\theta) d t\right.\right. \\
& \left.+\int_{\frac{1}{2}}^{1-\theta} t^{s}(1-t)(1-\theta-t) d t+\int_{1-\theta}^{1} t^{s}(1-t)(\theta+t-1) d t\right)\left|f^{\prime \prime}(a)\right|^{q} \\
& +\left(\int_{0}^{\theta} t(1-t)^{s}(\theta-t) d t+\int_{\theta}^{\frac{1}{2}} t(1-t)^{s}(t-\theta) d t\right. \\
& \left.\left.+\int_{\frac{1}{2}}^{1-\theta}(1-t)^{s+1}(1-\theta-t) d t+\int_{1-\theta}^{1}(1-t)^{s+1}(\theta+t-1) d t\right)\left|f^{\prime \prime}(b)\right|^{q}\right] \\
= & \frac{(b-a)^{2}}{2}\left(\frac{8 \theta^{3}-3 \theta+1}{24}\right)^{1-\frac{1}{q}}\left\{\left[\frac{2 \theta^{s+3}}{(s+2)(s+3)}\right.\right. \\
& \left.+\frac{2(1-\theta)^{s+2}[(s+1) \theta+2]+(s+3) \theta-2}{(s+1)(s+2)(s+3)} \frac{1-\theta}{2^{s+1}(s+1)(s+2)}\right] \\
& \left.\times\left(\left|f^{\prime \prime}(a)\right|^{q}+\left|f^{\prime \prime}(b)\right|^{q}\right)\right\}^{\frac{1}{q}},
\end{aligned}
$$

where

$$
\begin{aligned}
& \int_{0}^{1}|k(t)| d t=\int_{0}^{\frac{1}{2}}\left|\frac{1}{2} t(t-\theta)\right| d t+\int_{\frac{1}{2}}^{1}\left|\frac{1}{2}(1-t)(1-\theta-t)\right| d t \\
& =\frac{1}{2}\left[\int_{0}^{\theta} t(\theta-t) d t+\int_{\theta}^{\frac{1}{2}} t(t-\theta) d t\right. \\
& \left.+\int_{\frac{1}{2}}^{1-\theta}(1-t)(1-\theta-t) d t+\int_{1-\theta}^{1}(1-t)(t-1+\theta) d t\right] \\
& =\frac{8 \theta^{3}-3 \theta+1}{24} \text {, } \\
& \int_{0}^{\theta} t^{s+1}(\theta-t) d t=\int_{1-\theta}^{1}(1-t)^{s+1}(\theta+t-1) d t=\frac{\theta^{s+3}}{(s+2)(s+3)}, \\
& \int_{0}^{\theta} t(1-t)^{s}(\theta-t) d t=\int_{1-\theta}^{1}(1-t)(\theta+t-1) t^{s} d t \\
& =\frac{(1-\theta)^{s+2}[(s+1) \theta+2]+(s+3) \theta-2}{(s+1)(s+2)(s+3)}, \\
& \int_{\theta}^{\frac{1}{2}} t^{s+1}(t-\theta) d t=\int_{\frac{1}{2}}^{1-\theta}(1-t)^{s+1}(1-\theta-t) d t \\
& =\frac{(2 \theta)^{s+3}-2(s+3) \theta+s+2}{2^{s+3}(s+2)(s+3)},
\end{aligned}
$$

and

$$
\begin{aligned}
\int_{\theta}^{\frac{1}{2}} t(1-t)^{s}(t-\theta) d t & =\int_{\frac{1}{2}}^{1-\theta}(1-t)(1-\theta-t) t^{s} d t \\
& =\frac{(1-\theta)^{s+2}[(s+1) \theta+2]}{(s+1)(s+2)(s+3)}+\frac{2(s+3)^{2} \theta-s^{2}-7 s-14}{2^{s+3}(s+1)(s+2)(s+3)}
\end{aligned}
$$


In case $0 \leq \theta \leq \frac{1}{2}$, by Lemma 2 and using the Hölder inequality, we have

$$
\begin{aligned}
\left|\frac{1}{b-a} \int_{a}^{b} f(t) d t-(1-\theta) f\left(\frac{a+b}{2}\right)-\theta \frac{f(a)+f(b)}{2}\right| & \\
\leq & (b-a)^{2} \int_{0}^{1}|k(t)|\left|f^{\prime \prime}(t a+(1-t) b)\right| d t \\
\leq & (b-a)^{2}\left[\int_{0}^{1}|k(t)| d t\right]^{1-\frac{1}{q}}\left[\left.\int_{0}^{1}|k(t)| f^{\prime \prime}(t a+(1-t) b)\right|^{q} d t\right]^{\frac{1}{q}} \\
\leq & (b-a)^{2}\left(\frac{3 \theta-1}{24}\right)^{1-\frac{1}{q}}\left(\int_{0}^{1}|k(t)|\left[t^{s}\left|f^{\prime \prime}(a)\right|^{q}++(1-t)^{s}\left|f^{\prime \prime}(b)\right|^{q}\right] d t\right)^{\frac{1}{q}} \\
= & \frac{(b-a)}{2}\left(\frac{3 \theta-1}{24}\right)^{1-\frac{1}{q}}\left[\left(\int_{0}^{\frac{1}{2}} t^{s+1}|t-\theta| d t+\int_{\frac{1}{2}}^{1} t^{s}(1-t)|1-\theta-t| d t\right)\left|f^{\prime \prime}(a)\right|^{q}\right. \\
& \left.+\left(\int_{0}^{\frac{1}{2}} t(1-t)^{s}|t-\theta| d t+\int_{\frac{1}{2}}^{1}(1-t)^{s+1}|1-\theta-t| d t\right)\left|f^{\prime \prime}(b)\right|^{q}\right] \\
= & \frac{(b-a)}{2}\left(\frac{3 \theta-1}{24}\right)^{1-\frac{1}{q}}\left[\left(\int_{0}^{\frac{1}{2}} t^{s+1}(\theta-t) d t+\int_{\frac{1}{2}}^{1} t^{s}(1-t)(\theta+t-1) d t\right)\left|f^{\prime \prime}(a)\right|^{q}\right. \\
& \left.+\left(\int_{0}^{\frac{1}{2}} t(1-t)^{s}(\theta-t) d t+\int_{\frac{1}{2}}^{1}(1-t)^{s+1}(\theta+t-1) d t\right)\left|f^{\prime \prime}(b)\right|^{q}\right] \\
= & \frac{(b-a)^{2}}{2}\left(\frac{3 \theta-1}{24}\right)^{1-\frac{1}{q}}\left\{\left[\frac{(s+3) \theta-2}{(s+1)(s+2)(s+3)}+\frac{1-\theta}{2^{s+1}(s+1)(s+2)}\right]\right. \\
& \left.\times\left(\left|f^{\prime \prime}(a)\right|^{q}+\left|f^{\prime \prime}(b)\right|^{q}\right)\right\}^{\frac{1}{q}},
\end{aligned}
$$

where

$$
\begin{aligned}
& \begin{aligned}
\int_{0}^{1}|k(t)| d t & =\int_{0}^{\frac{1}{2}}\left|\frac{1}{2} t(t-\theta)\right| d t+\int_{\frac{1}{2}}^{1}\left|\frac{1}{2}(1-t)(1-\theta-t)\right| d t \\
& =\frac{1}{2}\left[\int_{0}^{\frac{1}{2}} t(\theta-t) d t+\int_{\frac{1}{2}}^{1}(1-t)(\theta+t-1) d t\right] \\
& =\frac{3 \theta-1}{24}
\end{aligned} \\
& \int_{0}^{\frac{1}{2}} t^{s+1}(\theta-t) d t=\int_{\frac{1}{2}}^{1}(1-t)^{s+1}(\theta+t-1) d t=\frac{(2 s+6) \theta-s-2}{2^{s+3}(s+2)(s+3)}
\end{aligned}
$$

and

$$
\begin{aligned}
\int_{0}^{\frac{1}{2}} t(1-t)^{s}(\theta-t) d t & =\int_{\frac{1}{2}}^{1}(1-t)(\theta+t-1) t^{s} d t \\
& =\frac{2^{s+3}[(s+3) \theta-2]-2(s+3)^{2} \theta+s^{2}+7 s+14}{2^{s+3}(s+1)(s+2)(s+3)} .
\end{aligned}
$$

The proof is thus completed. 
Remark 5 If we take $\theta=0$ in (28), then we get a midpoint type inequality

$$
\begin{aligned}
& \left|\frac{1}{b-a} \int_{a}^{b} f(t) d t-f\left(\frac{a+b}{2}\right)\right| \\
& \quad \leq \frac{(b-a)^{2}}{2}\left(\frac{1}{24}\right)^{1-\frac{1}{q}}\left[\frac{2^{s+2}-s-3}{2^{s+1}(s+1)(s+2)(s+3)}\left(\left|f^{\prime \prime}(a)\right|^{q}+\left|f^{\prime \prime}(b)\right|^{q}\right)\right]^{\frac{1}{q}} .
\end{aligned}
$$

If we take $\theta=1$ in (29), then we get a trapezoid type inequality

$$
\begin{aligned}
& \left|\frac{1}{b-a} \int_{a}^{b} f(t) d t-\frac{f(a)+f(b)}{2}\right| \\
& \quad \leq \frac{(b-a)^{2}}{2}\left(\frac{1}{4}\right)^{1-\frac{1}{q}}\left[\frac{\left|f^{\prime \prime}(a)\right|^{q}+\left|f^{\prime \prime}(b)\right|^{q}}{(s+2)(s+3)}\right]^{\frac{1}{q}} .
\end{aligned}
$$

If we take $\theta=\frac{1}{3}$ in (28), then we get a Simpson type inequality

$$
\begin{aligned}
& \mid \frac{1}{b-a} \int_{a}^{b} f(t) d t-\frac{f(a)+4 f\left(\frac{a+b}{2}\right)+f(b)}{6} \mid \\
& \leq \frac{(b-a)^{2}}{2}\left(\frac{1}{81}\right)^{1-\frac{1}{q}} \\
& \quad \times\left[\frac{2^{s+4}(s+1)+2^{2 s+6}(s+7)-6^{s+2}(6-2 s)-8(s+3) 3^{s+2}}{6^{s+3}(s+1)(s+2)(s+3)}\left(\left|f^{\prime \prime}(a)\right|^{q}+\left|f^{\prime \prime}(b)\right|^{q}\right)\right]^{\frac{1}{q}} .
\end{aligned}
$$

If we take $\theta=\frac{1}{2}$ in (28) or (29), then we get an averaged midpoint-trapezoid type inequality

$$
\begin{aligned}
& \left|\frac{1}{b-a} \int_{a}^{b} f(t) d t-\frac{1}{4}\left[f(a)+2 f\left(\frac{a+b}{2}\right)+f(b)\right]\right| \\
& \quad \leq \frac{(b-a)^{2}}{2}\left(\frac{1}{48}\right)^{1-\frac{1}{q}}\left[\frac{2^{s+1}(s-1)+s+3}{2^{s+2}(s+1)(s+2)(s+3)}\left(\left|f^{\prime \prime}(a)\right|^{q}+\left|f^{\prime \prime}(b)\right|^{q}\right)\right]^{\frac{1}{q}} .
\end{aligned}
$$

Corollary 2 Let $I \subset[0, \infty), f: I \rightarrow \mathbf{R}$ be a twice differentiable function on $I^{\circ}$ such that $f^{\prime \prime} \in L^{1}[a, b]$, where $a, b \in I$ with $a<b$. If $\left|f^{\prime \prime}\right|$ is s-convex in the second sense on $[a, b]$ for some fixed $s \in(0,1]$, then the following inequalities hold:

$$
\begin{aligned}
& \left|\frac{1}{b-a} \int_{a}^{b} f(x) d x-(1-\theta) f\left(\frac{a+b}{2}\right)-\theta \frac{f(a)+f(b)}{2}\right| \\
& \quad \leq \frac{(b-a)^{2}}{2}\left[\frac{2 \theta^{s+3}}{(s+2)(s+3)}\right. \\
& \left.\quad+\frac{2(1-\theta)^{s+2}((s+1) \theta+2)+(s+3) \theta-2}{(s+1)(s+2)(s+3)}-\frac{1-\theta}{2^{s+1}(s+1)(s+2)}\right]\left(\left|f^{\prime \prime}(a)\right|+\left|f^{\prime \prime}(b)\right|\right)
\end{aligned}
$$


for $0 \leq \theta \leq \frac{1}{2}$ and

$$
\begin{aligned}
& \left|\frac{1}{b-a} \int_{a}^{b} f(x) d x-(1-\theta) f\left(\frac{a+b}{2}\right)-\theta \frac{f(a)+f(b)}{2}\right| \\
& \quad \leq \frac{(b-a)^{2}}{2}\left[\frac{(s+3) \theta-2}{(s+1)(s+2)(s+3)}+\frac{1-\theta}{2^{s+1}(s+1)(s+2)}\right]\left(\left|f^{\prime \prime}(a)\right|+\left|f^{\prime \prime}(b)\right|\right)
\end{aligned}
$$

for $\frac{1}{2} \leq \theta \leq 1$.

Proof Inequalities (34) and (35) are immediate by setting $q=1$ in (28) and (29) of Theorem 4.

Remark 6 If we take $\theta=0$ in (34), then we get a midpoint type inequality

$$
\left|\frac{1}{b-a} \int_{a}^{b} f(t) d t-f\left(\frac{a+b}{2}\right)\right| \leq \frac{2^{s+2}-s-3}{2^{s+2}(s+1)(s+2)(s+3)}(b-a)^{2}\left(\left|f^{\prime \prime}(a)\right|+\left|f^{\prime \prime}(b)\right|\right) .
$$

If we take $\theta=1$ in (35), then we get a trapezoid type inequality

$$
\left|\frac{1}{b-a} \int_{a}^{b} f(t) d t-\frac{f(a)+f(b)}{2}\right| \leq \frac{1}{2(s+2)(s+3)}(b-a)^{2}\left(\left|f^{\prime \prime}(a)\right|+\left|f^{\prime \prime}(b)\right|\right) .
$$

If we take $\theta=\frac{1}{3}$ in (34), then we get a Simpson type inequality

$$
\begin{aligned}
& \left|\frac{1}{b-a} \int_{a}^{b} f(t) d t-\frac{1}{6}\left[f(a)+4 f\left(\frac{a+b}{2}\right)+f(b)\right]\right| \\
& \leq \frac{(s+1) 2^{s+3}+(s+7) 2^{2 s+5}-(3-s) 6^{s+2}-4(s+3) 3^{s+2}}{6^{s+3}(s+1)(s+2)(s+3)} \\
& \quad \times(b-a)^{2}\left(\left|f^{\prime \prime}(a)\right|+\left|f^{\prime \prime}(b)\right|\right) .
\end{aligned}
$$

If we take $\theta=\frac{1}{2}$ in (34) or (35), then we get an averaged midpoint-trapezoid type inequality

$$
\begin{aligned}
& \left|\frac{1}{b-a} \int_{a}^{b} f(t) d t-\frac{1}{4}\left[f(a)+2 f\left(\frac{a+b}{2}\right)+f(b)\right]\right| \\
& \quad \leq \frac{s+3-2^{s+1}(1-s)}{2^{s+3}(s+1)(s+2)(s+3)}(b-a)^{2}\left(\left|f^{\prime \prime}(a)\right|+\left|f^{\prime \prime}(b)\right|\right) .
\end{aligned}
$$

Remark 7 If we put $M=\sup _{x \in[a, b]}\left|f^{\prime \prime}\right|$ in (36)-(39), then we have

$$
\begin{aligned}
& \left|\frac{1}{b-a} \int_{a}^{b} f(t) d t-f\left(\frac{a+b}{2}\right)\right| \leq \frac{M\left(2^{s+2}-s-3\right)(b-a)^{2}}{2^{s+1}(s+1)(s+2)(s+3)} \\
& \left|\frac{1}{b-a} \int_{a}^{b} f(t) d t-\frac{f(a)+f(b)}{2}\right| \leq \frac{M(b-a)^{2}}{(s+2)(s+3)} \\
& \left|\frac{1}{b-a} \int_{a}^{b} f(t) d t-\frac{1}{6}\left[f(a)+4 f\left(\frac{a+b}{2}\right)+f(b)\right]\right| \\
& \quad \leq \frac{(s+1) 2^{s+3}+(s+7) 2^{2 s+5}-(3-s) 6^{s+2}-4(s+3) 3^{s+2}}{3(s+1)(s+2)(s+3) 6^{s+2}} M(b-a)^{2}
\end{aligned}
$$


and

$$
\begin{aligned}
& \left|\frac{1}{b-a} \int_{a}^{b} f(t) d t-\frac{1}{4}\left[f(a)+2 f\left(\frac{a+b}{2}\right)+f(b)\right]\right| \\
& \leq \frac{s+3-2^{s+1}(1-s)}{2^{s+2}(s+1)(s+2)(s+3)} M(b-a)^{2} .
\end{aligned}
$$

Remark 8 If we further take $s=1$ in (40)-(43), i.e., for functions $f$ with convex $\left|f^{\prime \prime}\right|$, then we recapture inequalities (23)-(26).

\section{Applications to special means}

We consider the means for arbitrary positive numbers $\alpha, \beta(\alpha \neq \beta)$ as follows:

(1) The arithmetic mean

$$
A(\alpha, \beta)=\frac{\alpha+\beta}{2}
$$

(2) The geometric mean

$$
G(\alpha, \beta)=\sqrt{\alpha \beta}
$$

(3) The harmonic mean

$$
H(\alpha, \beta)=\frac{2 \alpha \beta}{\alpha+\beta}
$$

(4) The logarithmic mean

$$
L(\alpha, \beta)=\frac{\beta-\alpha}{\ln \beta-\ln \alpha} .
$$

(5) The generalized log-mean

$$
L_{p}(\alpha, \beta)=\left[\frac{\beta^{p+1}-\alpha^{p+1}}{(p+1)(\beta-\alpha)}\right]^{\frac{1}{p}}, \quad p \neq-1,0
$$

(6) The identric mean

$$
I(\alpha, \beta)=\frac{1}{e}\left(\frac{\beta^{\beta}}{\alpha^{\alpha}}\right) \frac{1}{\beta-\alpha} .
$$

Proposition 1 Let $0<a<b$ and $s \in(0,1)$. Then we have

$$
\begin{aligned}
& \left|A^{s}(a, b)-L_{s}^{s}(a, b)\right| \leq \frac{s(1-s)(b-a)^{2}}{24 a^{2-s}}, \\
& \left|A\left(a^{s}, b^{s}\right)-L_{s}^{s}(a, b)\right| \leq \frac{s(1-s)(b-a)^{2}}{12 a^{2-s}}, \\
& \left|\frac{2 A^{s}(a, b)+A\left(a^{s}, b^{s}\right)}{3}-L_{s}^{s}(a, b)\right| \leq \frac{s(1-s)(b-a)^{2}}{81 a^{2-s}},
\end{aligned}
$$


and

$$
\left|\frac{A^{s}(a, b)+A\left(a^{s}, b^{s}\right)}{2}-L_{s}^{s}(a, b)\right| \leq \frac{s(1-s)(b-a)^{2}}{48 a^{2-s}} .
$$

Proof The assertion follows from applying inequalities (23)-(26) to the mapping $f(x)=x^{s}$, $x \in[a, b]$, which implies that $\left|f^{\prime \prime}(x)\right|=s(1-s) x^{s-2}$ is convex on $[a, b]$, and we may take $M=\frac{s(1-s)}{a^{2-s}}$.

Proposition 2 Let $0<a<b$. Then we have

$$
\begin{aligned}
& \left|A^{-1}(a, b)-L^{-1}(a, b)\right| \leq \frac{(b-a)^{2}}{12 a^{3}} \\
& \left|H^{-1}(a, b)-L^{-1}(a, b)\right| \leq \frac{(b-a)^{2}}{6 a^{3}} \\
& \left|\frac{2 A^{-1}(a, b)+H^{-1}(a, b)}{3}-L^{-1}(a, b)\right| \leq \frac{2(b-a)^{2}}{81 a^{3}}
\end{aligned}
$$

and

$$
\left|\frac{A^{-1}(a, b)+H^{-1}(a, b)}{2}-L^{-1}(a, b)\right| \leq \frac{(b-a)^{2}}{24 a^{3}} .
$$

Proof The assertion follows from applying inequalities (23)-(26) to the mapping $f(x)=\frac{1}{x}$, $x \in[a, b]$, which implies that $\left|f^{\prime \prime}(x)\right|=\frac{2}{x^{3}}$ is convex on $[a, b]$, and we may take $M=\frac{2}{a^{3}}$.

Proposition 3 Let $a, b \in \mathbf{R}, 0<a<b$. Then we have

$$
\begin{aligned}
& |\ln A(a, b)-\ln I(a, b)| \leq \frac{(b-a)^{2}}{24 a^{2}} \\
& |\ln G(a, b)-\ln I(a, b)| \leq \frac{(b-a)^{2}}{12 a^{2}} \\
& \left|\frac{2 \ln A(a, b)+\ln G(a, b)}{3}-\ln I(a, b)\right| \leq \frac{(b-a)^{2}}{81 a^{2}}
\end{aligned}
$$

and

$$
\left|\frac{\ln A(a, b)+\ln G(a, b)}{2}-\ln I(a, b)\right| \leq \frac{(b-a)^{2}}{48 a^{2}} .
$$

Proof The assertion follows from applying inequalities (23)-(26) to the mapping $f(x)=$ $\ln x, x \in[a, b]$, which implies that $\left|f^{\prime \prime}(x)\right|=\frac{1}{x^{2}}$ is convex on $[a, b]$, and we may take $M=\frac{1}{a^{2}}$. 
References

1. Hudzik, H, Maligranda, L: Some remarks on s-convex functions. Aequ. Math. 17(2), 100-111 (1994)

2. Özdemir, ME, Yildiz, Ç, Akdemir, AO, Set, E: On some inequalities for s-convex functions and applications. J. Inequal. Appl. 2013,333 (2013)

3. Liu, Z: A note on Ostrowski type inequalities related to some s-convex functions in the second sense. Bull. Korean Math. Soc. 49(4), 775-785 (2012)

4. Sarikaya, MZ, Aktan, N: On the generalization of some integral inequalities and their applications. arXiv:1005.2879v1 [math.CA] (17 May 2010)

Submit your manuscript to a SpringerOpen ${ }^{\circ}$ journal and benefit from:

- Convenient online submission

- Rigorous peer review

- Immediate publication on acceptance

- Open access: articles freely available online

- High visibility within the field

- Retaining the copyright to your article 